\title{
Análisis de la victimización/agresión sexual a través de las tipologías de adhesión al doble estándar sexual en población general
}

\author{
Cristina Vílchez-Jaén, Ana Álvarez-Muelas y Juan Carlos Sierra* \\ Centro de Investigación Mente, Cerebro y Comportamiento, Universidad de Granada, España \\ - Recibido: 03 - 12 - 2021 . Aceptado:14 - 12 - 2021
}

RESUMEN. Antecedentes/Objetivo. El doble estándar sexual (DES) (i.e., actitud que implica diferentes criterios para evaluar comportamientos sexuales de hombres y mujeres) se ha relacionado con la victimización y agresión sexual. Se examina el efecto del sexo, la tipología de adhesión al DES (igualitaria, favorable al hombre y favorable a la mujer) en los ámbitos de la libertad y del recato sexual, y de la interacción sexo x tipología sobre distintas categorías de victimización y agresión sexual. Método: En 716 adultos españoles heterosexuales (264 hombres y 452 mujeres), de 18 a 66 años, se evaluaron variables sociodemográficas, DES y experiencias informadas de victimización y agresión sexual. Resultados: En victimización sexual se encontró un efecto significativo del sexo con incrementos del 21-27.4\% por el hecho de ser mujer. En agresión sexual, el efecto significativo del sexo implicó incrementos del 12.4-13.9\% en intentos de violación y violación por ser hombre. Los hombres con tipología de adhesión al DES favorable al hombre informaron mayor número de agresiones de violación, con respecto al resto de tipologías. Conclusiones: El empleo de las tipologías de adhesión al DES contribuye al estudio de la relación de esta actitud con la victimización y agresión sexual.

PALABRAS CLAVE: Doble Estándar Sexual, Victimización Sexual, Agresión Sexual, Diferencias Sexuales.

Analysis of sexual victimization/aggression through typologies of adherence to sexual double standard in general population

ABSTRACT. Background/Objective: Sexual double standard (SDS) (i.e., the attitude involving different criteria for evaluating sexual behaviors of men and women) has been related to sexual victimization and aggression. It was examined the effect of sex, the typology of adherence to SDS in the sexual freedom and sexual shyness areas (egalitarian, man-favorable, and woman-favorable), and of the sex $x$ SDS type interaction on different categories of sexual victimization and sexual aggression. Methods: In a sample of 716 Spanish heterosexual adults (264 men and 452 women), aged 18 to 66 years, sociodemographic variables, SDS and reported experiences of sexual victimization and aggression were assessed. Results: In sexual victimization, a significant effect of sex was found with increases of $21-27.4 \%$ for being a woman. In sexual aggression, the significant effect of sex implied increases of $12.4-13.9 \%$ in attempted rape and rape for being male. Men with man-favorable SDS typology reported higher numbers of rape assaults, relative to all other typologies. Conclusions: The use of the SDS typologies of adherence contributes to the study of the relationship of this attitude with sexual victimization and aggression.

KEYWORDS: Sexual Double Standard, Sexual Victimization, Sexual Aggression, Sexual Differences.

La European Union Agency for Fundamental Rights (2014) estima que 3,7 millones de mujeres mayores de edad pertenecientes a la

\footnotetext{
${ }^{*}$ Correspondencia: Juan Carlos Sierra

Facultad de Psicología. Campus Universitario de Cartuja, s/n. 18011

Granada (España).

E-mail: jcsierra@ugr.es
}

Unión Europea fueron víctimas de agresión sexual durante los 12 meses previos a la encuesta, siendo en el $97 \%$ de los casos el autor un hombre. Según la Oficina Europea de Estadística (2021), en Europa, desde 2009 a 2017 el número de víctimas de agresión sexual se ha incrementado un $57 \%$ y el de violaciones un $123 \%$. Los datos registrados indican que, en 2017, 156.120 personas fueron víctimas de 
agresión sexual (83\% mujeres) y 102.441 de violación ( $90 \%$ mujeres); sin embargo, solo se han registrado 20.034 personas condenadas por agresión sexual y 8.829 por violación (99\% hombres) (Oficina Europea de Estadística, 2021). En España, el Ministerio del Interior registró en 2019, 15.319 delitos contra la libertad sexual, correspondiéndose el 78\% a delitos de agresión y abuso sexual, siendo el $85 \%$ víctimas del sexo femenino. Estos delitos se han incrementado un 83\% desde 2013 (Ministerio del Interior, 2019).

El amplio número de delitos sexuales y su aumento en los últimos años ha suscitado el interés por su casuística (e.g., Garrido-Macías et al., 2020; Wilson et al., 2015). Diversos estudios relacionan la agresión sexual con actitudes sexistas (Álvarez-Muelas et al., 2020; Malonda et al., 2017; Martínez-Gómez et al., 2021). En esta línea, destaca la actitud del doble estándar sexual (DES), que alude a la evaluación de un comportamiento sexual de forma diferente en función de si lo realiza un hombre o una mujer (Fasula et al., 2014). Tradicionalmente, el DES asume mayor libertad sexual para el hombre que para la mujer (Álvarez-Muelas et al., 2019; Guo, 2019; Sánchez-Fuentes et al., 2020; Sierra et al., 2018). El DES que resulta favorable para el hombre se ha relacionado con agresividad sexual del hombre hacia la mujer (Moyano et al., 2017; Noriega et al., 2020; Sierra, Gutiérrez-Quintanilla et al., 2009; Teitelman et al., 2013) y con el apoyo de actitudes a favor de la violación hacia la mujer (Jamshed \& Kamal, 2019; Lee et al., 2010; Mittal et al., 2017; Moyano et al., 2017; Sierra, Costa, \& Ortega, 2009; Wanfield, 2018), constituyendo un predictor de las mismas (Sierra et al., 2010). El DES también se ha relacionado con la victimización sexual de la mujer (Dunn et al., 2014; Koon-Magnin \& Ruback, 2012; Lee et al., 2010; Sierra et al., 2010) y una mayor dificultad para el reconocimiento de la violencia sexual, tanto en hombres como en mujeres (Kim et al., 2019).

Recientemente, se ha propuesto el estudio del DES distinguiendo dos ámbitos de comportamientos sexuales: libertad y recato sexual (Sierra et al., 2018). La libertad sexual se define como "el reconocimiento y aprobación de un beneficio a hombres y mujeres, de tener relaciones sexuales libremente respetando los derechos" (Álvarez-Muelas et al., 2021, p. $2)$, mientras que el recato sexual se refiere al "reconocimiento y la aprobación de la voluntad de hombres y mujeres de manifestar decoro, castidad y continencia en las relaciones sexuales" (Álvarez-Muelas et al., 2021, p. 2). Además, las diferentes tipologías de adhesión al DES permiten describir distintas formas de expresión que adopta esta actitud (Álvarez-Muelas et al., 2021): favorable al hombre, favorable a la mujer e igualitaria. La favorable al hombre describe a personas que apoyan más libertad y menos recato sexual para hombres que para mujeres; la favorable a la mujer se refiere a personas que apoyan más libertad y menos recato sexual para mujeres que para hombres; finalmente, la igualitaria incluye a personas que apoyan la misma libertad y recato sexual para hombres que para mujeres (Álvarez-Muelas et al., 2021).

Hasta la fecha no se ha analizado la relación de la agresión y victimización sexual con las distintas tipologías de adhesión al DES. Por ello, el objetivo del presente estudio es examinar el efecto del sexo, de la tipología de adhesión al DES en los ámbitos de libertad y recato sexual (igualitaria, favorable al hombre y favorable a la mujer), y de la interacción de ambos factores, en distintas categorías de victimización y agresión sexual (contacto sexual, coerción, intento de violación y violación). De acuerdo con la evidencia previa, se espera encontrar que: (a) los hombres, en comparación con las mujeres, informarán menor número de experiencias de victimización sexual y mayor número de agresiones sexuales; y (b) la tipología de adhesión al DES favorable al hombre se asociará a mayor número de experiencias de victimización y agresión sexual.

\section{MÉTODO}

\section{-PARTICIPANTES}

Mediante muestreo no probabilístico se obtuvo, entre la población general española, una muestra de 716 adultos heterosexuales 
(264 hombres y 452 mujeres) de 18 a 66 años. Las características sociodemográficas de los participantes se encuentran en la Tabla 1.

\section{- INSTRUMENTOS}

- Cuestionario Sociodemográfico. Recoge información acerca del sexo, edad, nacionalidad, orientación sexual, nivel educativo, nivel económico, relación de pareja y número de parejas sexuales.

- Versión española del Sexual Experiences Survey (Koss et al., 2007). Consta de dos subescalas formadas por diez ítems cada una, contestados en una escala Likert, desde 0 (nunca) hasta 5 (5 o más veces). Se responde como autor de agresiones sexuales (subescala Agresión sexual) y como víctima de ellas (subescala Victimización sexual) después de los 14 años en relación a cuatro categorías: contactos sexuales (experiencias de contactos sexuales como besos o caricias, sin que exista un intento de penetración, provocadas por presión verbal, abuso de autoridad, amenazas o uso de la fuerza), coerción sexual (coito con una persona mediante el uso de presiones verbales o abuso de autoridad), intento de violación (intento de mantener una relación coital con alguien que no lo desea, mediante el uso de alcohol o drogas, amenazas o uso de fuerza) y violación (contacto sexual con penetración con alguien que no lo desea mediante el uso de alcohol o drogas, amenazas o fuerza física) (Sierra et al., 2014).

Tabla 1

Características sociodemográficas de los participantes

\begin{tabular}{|c|c|c|c|}
\hline & $\begin{array}{c}\text { Total } \\
(N=716)\end{array}$ & $\begin{array}{l}\text { Hombres } \\
(n=264)\end{array}$ & $\begin{array}{c}\text { Mujeres } \\
(n=452)\end{array}$ \\
\hline Edad M (DT) & $31.62(10.36)$ & $33.26(9.37)$ & 30.67 (10.79) \\
\hline \multicolumn{4}{|l|}{ Nivel educativo $n$ [\%] } \\
\hline Sin estudios & $3(0.40 \%)$ & $1(0.40 \%)$ & $2(0.40 \%)$ \\
\hline Estudios Primarios & $17(2.40 \%)$ & $6(2.30 \%)$ & $11(2.40 \%)$ \\
\hline Estudios Secundarios & 122 (17\%) & $75(28.40 \%)$ & 47 (10.40\%) \\
\hline Estudios Universitarios & $469(65.50 \%)$ & $135(51.10 \%)$ & $334(73.90 \%)$ \\
\hline Otros & $103(14.40 \%)$ & $45(17 \%)$ & $58(12.80 \%)$ \\
\hline \multicolumn{4}{|l|}{ Ingresos económicos n [\%] } \\
\hline Hasta $499 €$ & 95 (17\%) & $21(9.10 \%)$ & $74(22.70 \%)$ \\
\hline 500 a $1.499 €$ & $188(33.70 \%)$ & $54(23.30 \%)$ & $134(41.10 \%)$ \\
\hline 1.500 a $1.999 €$ & 85 (15.20\%) & $36(15.50 \%)$ & 49 (15\%) \\
\hline 2.000 a $2.499 €$ & $143(25.60 \%)$ & $92(39.70 \%)$ & $51(15.60 \%)$ \\
\hline 2.500 a $2.999 €$ & $32(5.70 \%)$ & $20(3.40 \%)$ & $12(0.90 \%)$ \\
\hline 3.000 a $4.999 €$ & $11(2 \%)$ & $8(0.40 \%)$ & $3(0.90 \%)$ \\
\hline $5.000 €$ o más & $4(0.70 \%)$ & $1(0.40 \%)$ & $3(0.90 \%)$ \\
\hline Tiene pareja actualmente $n$ (\%) & $524(73.20 \%)$ & $196(74.20 \%)$ & $328(72.60 \%)$ \\
\hline Número parejas sexuales $M(D T)$ & $9.82(14.92)$ & $13.16(20.69)$ & $7.88(9.70)$ \\
\hline Meses de relación con la pareja actual M (DT) & $82.93(110.70)$ & $94.20(109.44)$ & $76.35(111.03)$ \\
\hline
\end{tabular}


Moyano y Sierra (2014) informan un alfa de Cronbach de .82 en hombres y .75 en mujeres. En este estudio, los valores alfa de Cronbach fueron .52 para la subescala de Agresión sexual y .68 para Victimización sexual.

- Versión española de la Sexual Double Standard Scale (Muehlenhard \& Quackenbush, 2011) de Sierra et al. (2018). Consta de 16 ítems respondidos en una escala Likert, desde 0 (muy en desacuerdo) hasta 3 (muy de acuerdo), agrupados en dos factores: Aceptación de la libertad sexual y Aceptación del recato sexual. Cada factor está formado por ocho ítems paralelos, es decir, cuatro pares de ítems. En cada par, un ítem se refiere a un comportamiento sexual atribuido al hombre y el otro al mismo comportamiento atribuido a la mujer. Las puntuaciones de los pares de cada factor permiten obtener el Índice de Doble Estándar para Libertad Sexual (IDE-LS) y el Índice de Doble Estándar para Recato Sexual (IDE-RS). La escala mostró una adecuada fiabilidad de consistencia interna (.84 en Aceptación de la libertad sexual y .87 en Aceptación del recato sexual) (Sierra et al., 2018). En este estudio, se obtuvieron valores de .76 para Aceptación de la libertad sexual y .84 para Aceptación del recato sexual.

\section{-PROCEDIMIENTO}

La encuesta fue diseñada mediante el software LimeSurvey ${ }^{\circledR}$ ubicado en los servidores de la Universidad de Granada y difundida a través de redes sociales (Facebook ${ }^{\circledR}$, Instagram ${ }^{\circledR}$, WhatsApp $\left.{ }^{\circledR}\right)$ y por correo electrónico. Como medidas de control se revisaron las direcciones IP y el acceso a la encuesta tenía una pregunta de seguridad que consistía en una sencilla operación aritmética. Además, un examen minucioso de datos permitió descartar casos con respuestas repetidas, incongruentes o patrones anómalos. La encuesta iba acompañada de un consentimiento informado, que garantizaba a los encuestados el anonimato y la máxima confidencialidad. La participación era completamente voluntaria. El estudio recibió previamente la aprobación del Comité de Ética en Investigación con Humanos de la Universidad de Granada.

\section{-ANÁLISIS DE DATOS}

La imputación de los valores faltantes se realizó a través del paquete missForest (versión 1.4; Stekhoven \& Bühlmann, 2011). Se realizó la categorización de los participantes entre las tipologías del DES para el ámbito de la libertad sexual y para el ámbito del recato sexual, en función de las puntuaciones en cada índice de doble estándar sexual. La tipología favorable al hombre incluye personas con puntuación positiva en el índice (entre +1 y +12 ). La tipología favorable a la mujer incluye personas con puntuación negativa en el índice (entre - 1 y -12). La tipología igualitaria incluye personas con puntuaciones de cero en el índice y en la diferencia para cada par de ítems que componen el índice.

El diseño del análisis de datos requiere de la ejecución de ANOVAs. No obstante, observamos falta de homogeneidad (prueba de Levene significativa) en algunas de las variables. La ausencia de homogeneidad de varianza cuando los grupos no son de igual tamaño (i.e., grupos mayor/grupo menor > 1.5) puede provocar desviaciones importantes en la significatividad de los resultados (Stevens, 1986). Por ello, se verificó, cuando la $p$ para $F$ era $<.05$ (significativa), el cumplimiento de tres criterios que salvaguardan de estas desviaciones (Mayorga et al., 2020): (a) que la $F$ teórica fuera menor que el valor empírico de $F$, (b) que la razón $(\beta / \alpha)$ entre el falso rechazo de la hipótesis nula y la falsa aceptación de la hipótesis nula fuera $\geq 1$, y (c) que la magnitud del tamaño del efecto estuviera en línea con la aceptación hipótesis nula ( $\geq 0.20)$. En todos los resultados con valores de $F$ significativos se cumplieron estos tres requisitos. Los contrastes a posteriori se ejecutaron, observada homogeneidad de varianza, con la corrección de Bonferroni, y C de Howell y Dunnett. El tamaño del efecto en las comparaciones de medias se obtuvo en d con la fórmula de Glass's cuando no había homogeneidad de varianza y la de Hedges con Ns de tamaños diferentes y se estimó la magnitud en el porcentaje de incremento (Fandiño et al., 2021). 


\section{RESULTADOS}

En primer lugar, se examinó la relación de la edad con la frecuencia de experiencias de victimizaciónyagresiónsexual, noencontrándose ninguna correlación significativa.

\section{-ÁMBITO DE LA LIBERTAD SEXUAL}

Ejecutados ANOVAs 2 (sexo: hombre y mujer) x 3 (tipología DES en el ámbito de la libertad sexual: igualitaria, favorable al hombre y favorable a la mujer) sobre la victimización sexual, los resultados mostraron (ver Tabla 2) para el factor sexo un efecto significativo en contacto sexual y coerción, pero no así para intento de violación y violación. En concreto, las mujeres informan de una mayor victimización de contacto sexual $(M=2.03)$ que los hombres $(M=1.12)$, con un tamaño del efecto $d=$ 0.43 , lo que implica un incremento del $21.0 \%$ asociado a ser mujer en la victimización de contacto sexual. Asimismo, las mujeres advierten de una mayor victimización de coerción $(M=1.28)$ que los hombres $(M=$ 0.54 ), con un tamaño del efecto $d=0.55$, lo que implica un incremento del $26.5 \%$ asociado a ser mujer en la victimización de coerción sexual. Por su parte, la tipología DES en el ámbito de la libertad sexual no tiene efectos sobre la victimización (i.e., no hay diferencias en ninguna de las categorías de victimización). Además, no se observó interacción significativa entre ambos factores, es decir, sexo y tipología DES en el ámbito de la libertad sexual son totalmente independientes en lo que a la victimización se refiere.

Asimismo, realizados ANOVAs 2 (sexo: hombre y mujer) x 3 (tipología DES en el ámbito de la libertad sexual: igualitaria, favorable al hombre y favorable a la mujer) sobre la

Tabla 2

Análisis del sexo, tipología DES en el ámbito de la libertad sexual e interacción de ambas variables en victimización sexual

\begin{tabular}{|c|c|c|c|c|}
\hline Variable (g.l.) & $F$ & $p$ & $d / \eta^{2} p$ & $1-\beta$ \\
\hline \multicolumn{5}{|l|}{ Victimización de contacto sexual } \\
\hline $\operatorname{Sexo}(1,637)$ & 11.28 & $<.001$ & 0.43 & .918 \\
\hline Tipología DES $(2,637)$ & 0.53 & .589 & .002 & .138 \\
\hline Sexo x Tipología DES $(2,637)$ & 0.72 & .487 & .002 & .172 \\
\hline \multicolumn{5}{|l|}{ Victimización de coerción } \\
\hline $\operatorname{Sexo}(1,640)$ & 13.83 & $<.001$ & 0.55 & .960 \\
\hline Tipología DES $(2,640)$ & 0.03 & .971 & .000 & .054 \\
\hline Sexo x Tipología DES $(2,640)$ & 0.47 & .625 & .001 & .127 \\
\hline \multicolumn{5}{|l|}{ Victimización de intento de violación } \\
\hline $\operatorname{Sexo}(1,639)$ & 2.01 & .156 & 0.10 & .294 \\
\hline Tipología DES $(2,639)$ & 0.66 & .517 & .002 & .161 \\
\hline Sexo x Tipología DES $(2,639)$ & 0.27 & .765 & .001 & .092 \\
\hline \multicolumn{5}{|l|}{ Victimización de violación } \\
\hline $\operatorname{Sexo}(1,637)$ & 0.48 & .488 & 0.06 & .107 \\
\hline Tipología DES $(2,637)$ & 0.32 & .729 & .001 & .100 \\
\hline Sexo x Tipología DES $(2,637)$ & 0.07 & .930 & .000 & .061 \\
\hline
\end{tabular}


Tabla 3

Análisis del sexo, tipología DES en el ámbito de la libertad sexual e interacción de ambas variables en victimización sexual

\begin{tabular}{|c|c|c|c|c|}
\hline Variable (g.l.) & $F$ & $p$ & $d / \eta^{2} p$ & $1-\beta$ \\
\hline \multicolumn{5}{|l|}{ Agresión de contacto sexual } \\
\hline $\operatorname{Sexo}(1,604)$ & 1.82 & .178 & 0.10 & .271 \\
\hline Tipología DES $(2,604)$ & 0.82 & .441 & .003 & .191 \\
\hline Sexo x Tipología DES $(2,604)$ & 2.00 & .137 & .007 & .413 \\
\hline \multicolumn{5}{|l|}{ Agresión de coerción } \\
\hline Sexo $(1,602)$ & 2.32 & .128 & 0.12 & .331 \\
\hline Tipología DES $(2,602)$ & 1.11 & .330 & .004 & .246 \\
\hline Sexo x Tipología DES $(2,602)$ & 0.96 & .383 & .003 & .217 \\
\hline \multicolumn{5}{|l|}{ Agresión de intento de violación } \\
\hline Sexo $(1,604)$ & 6.95 & .009 & 0.27 & .749 \\
\hline Tipología DES $(2,604)$ & 2.03 & .132 & .007 & .419 \\
\hline Sexo x Tipología DES $(2,604)$ & 2.48 & .084 & .008 & .499 \\
\hline \multicolumn{5}{|l|}{ Agresión de violación } \\
\hline $\operatorname{Sexo}(1,608)$ & 7.08 & .008 & 0.28 & .757 \\
\hline Tipología DES $(2,608)$ & 5.09 & .006 & .016 & .821 \\
\hline Sexo x Tipología DES $(2,608)$ & 5.09 & .006 & .016 & .821 \\
\hline
\end{tabular}

agresión sexual, mostraron (ver Tabla 3) un efecto significativo en intento de violación y violación para el factor sexo, y en violación para el factor tipología DES y para la interacción de sexo x tipología DES en el ámbito de libertad sexual. Sucintamente, los hombres advierten de un mayor número de intentos de violación $(M$ $=0.06)$ que las mujeres $(M=0.00)$, con un tamaño del efecto $d=0.27$, lo que implica un incremento del $13.4 \%$ asociado a ser hombre en la agresión de intento de violación; así como de violación (Ms $=0.02$ y 0.00 , para hombres y mujeres, respectivamente), con un tamaño del efecto $d=0.28$, lo que implica un incremento del $13.9 \%$ asociado a ser hombre en la agresión de violación. Por su parte, los contrastes a posteriori (C de Howell y Dunnett) para el factor tipología DES informan que la tipología favorable al hombre $(M=0.14)$ informa de mayor agresión de violación que la favorable a la mujer $(M=0.00)$, con un tamaño del efecto $d=0.21$, lo que implica un incremento del $10.4 \%$ asociado a la tipología favorable al hombre; y la igualitaria $(M=$ 0.00 ), con un tamaño del efecto $d=0.20$, lo que implica un incremento del $10.0 \%$ asociado a la tipología favorable al hombre. Para la interacción de sexo x tipología DES en el ámbito de la libertad sexual para la agresión sexual de violación, véase la Figura 1.

\section{-ÁMBITO DEL RECATO SEXUAL}

Los resultados de los ANOVAs 2 (sexo: hombre y mujer) x 3 (tipología DES en el ámbito del recato sexual: igualitaria, favorable al hombre y favorable a la mujer) sobre la victimización sexual pusieron de manifiesto (ver Tabla 4) para el factor sexo un efecto significativo en contacto sexual y coerción, no 
así para intento de violación y violación. Las mujeres informan de una mayor victimización de contacto sexual ( $M=2.04)$ que los hombres $(M=1.07)$, con un tamaño del efecto $d=0.46$, lo que implica un incremento en la victimización de contacto sexual del $22.4 \%$ asociado a ser mujer; así como mayor victimización de coerción ( $M s=1.30$ y 0.53 , respectivamente), con un tamaño del efecto $d$ $=0.57$, lo que implica un incremento en la victimización de coerción sexual del $27.4 \%$ asociado a ser mujer. La tipología DES en el ámbito del recato sexual no tiene efectos en la victimización. Tampoco se observa interacción significativa entre ambos factores, es decir, sexo y tipología DES en el ámbito del recato sexual son totalmente independientes en lo que a la victimización se refiere.
Finalmente, los resultados de los ANOVAs 2 (sexo: hombre y mujer) x 3 (tipología DES en el ámbito del recato sexual: igualitaria, favorable al hombre y favorable a la mujer) realizados para la agresión sexual evidenciaron (ver Tabla 5) un efecto significativo del sexo sobre intento de violación, siendo los hombres $(M=0.05)$ quienes informan más intentos que las mujeres $(M=0.00)$, con un tamaño del efecto $d=0.25$, lo que implica un incremento en la probabilidad de agresión de intento de violación del $12.4 \%$ asociado a ser hombre. En violación, se encuentra un efecto significativo para el factor tipología DES en el ámbito del recato sexual y para la interacción de sexo $\mathrm{x}$ tipología DES. Los contrastes a posteriori (C de Howell y Dunnett) para el factor tipología DES en el ámbito de recato sexual informan que la

Tabla 4

Análisis del sexo, tipología DES en el ámbito del recato sexual e interacción de ambas variables en victimización sexual

\begin{tabular}{|c|c|c|c|c|}
\hline Variable (g.l.) & $F$ & p & $d / \eta^{2} p$ & $1-\beta$ \\
\hline \multicolumn{5}{|l|}{ Victimización de contacto sexual } \\
\hline Sexo $(1,628)$ & 23.74 & $<.001$ & 0.46 & .998 \\
\hline Tipología DES $(2,628)$ & 1.57 & .210 & .005 & .333 \\
\hline Sexo x Tipología DES $(2,628)$ & 0.08 & .924 & .000 & .062 \\
\hline \multicolumn{5}{|l|}{ Victimización de coerción } \\
\hline $\operatorname{SexO}(1,633)$ & 25.75 & $<.001$ & 0.57 & .999 \\
\hline Tipología DES $(2,633)$ & 1.63 & .198 & .005 & .344 \\
\hline Sexo x Tipología DES $(2,633)$ & 0.11 & .897 & .000 & .067 \\
\hline \multicolumn{5}{|l|}{ Victimización de intento de violación } \\
\hline $\operatorname{Sexo}(1,632)$ & 1.14 & .286 & 0.09 & .187 \\
\hline Tipología DES $(2,632)$ & 0.56 & .571 & .002 & .143 \\
\hline Sexo x Tipología DES $(2,632)$ & 0.42 & .657 & .001 & .118 \\
\hline \multicolumn{5}{|l|}{ Victimización de violación } \\
\hline $\operatorname{Sexo}(1,630)$ & 1.21 & .271 & 0.09 & .196 \\
\hline Tipología DES $(2,630)$ & 0.34 & .716 & .001 & .104 \\
\hline Sexo x Tipología DES $(2,630)$ & 0.40 & .668 & .001 & .115 \\
\hline
\end{tabular}




\section{Tabla 5}

Análisis del sexo, tipología DES en el ámbito del recato sexual e interacción de ambas variables en agresión sexual

\begin{tabular}{|c|c|c|c|c|}
\hline Variable (g.l.) & $F$ & $p$ & $d / \eta^{2} p$ & $1-\beta$ \\
\hline \multicolumn{5}{|l|}{ Agresión de contacto sexual } \\
\hline Sexo $(1,596)$ & 0.06 & .810 & 0.00 & .057 \\
\hline Tipología DES $(2,596)$ & 0.53 & .591 & .002 & .137 \\
\hline Sexo x Tipología DES $(2,596)$ & 0.20 & .820 & .001 & .081 \\
\hline \multicolumn{5}{|l|}{ Agresión de coerción } \\
\hline Sexo $(1,596)$ & 1.79 & .182 & 0.10 & .266 \\
\hline Tipología DES $(2,596)$ & 0.82 & .441 & .003 & .191 \\
\hline Sexo x Tipología DES $(2,596)$ & 0.86 & .423 & .003 & .199 \\
\hline \multicolumn{5}{|l|}{ Agresión de intento de violación } \\
\hline Sexo $(1,599)$ & 5.68 & .017 & 0.25 & .662 \\
\hline Tipología DES $(2,599)$ & 1.36 & .258 & .005 & .293 \\
\hline Sexo x Tipología DES $(2,599)$ & 2.00 & .135 & .007 & .415 \\
\hline \multicolumn{5}{|l|}{ Agresión de violación } \\
\hline $\operatorname{Sexo}(1,602)$ & 3.62 & .058 & 0.18 & .475 \\
\hline Tipología DES $(2,602)$ & 3.08 & .047 & .010 & .593 \\
\hline Sexo x Tipología DES $(2,602)$ & 3.08 & .047 & .010 & .593 \\
\hline
\end{tabular}

tipología DES favorable al hombre $(M=0.05)$ advierte de mayor agresión de violación que la favorable a la mujer $(M=0.00)$, con un tamaño del efecto $d=0.20$, lo que implica un incremento del $10.0 \%$ asociado a la tipología favorable al hombre, y la igualitaria $(M=0.00)$, con un tamaño del efecto $d=$ 0.21 , lo que implica un incremento del $10.4 \%$ asociado a la tipología favorable al hombre. Para la interacción de sexo x tipología DES en el ámbito del recato sexual para la agresión sexual de violación, véase la Figura 1.

\section{DISCUSIÓN}

El objetivo de este estudio fue aportar evidencias acerca de la relación de la victimización y agresión sexual con las tipologías de adhesión al DES, empleando la Sexual Double
Stantard Scale (Muehlenhard \& Quackenbush, 2011; Sierra et al., 2018), escala que permite obtener diferentes tipologías de adhesión al DES (igualitaria, favorable al hombre y favorable a la mujer) en dos ámbitos de los comportamientos sexuales: libertad sexual y recato sexual. Para ello, se examinó en diferentes categorías de victimización y agresión sexual (i.e., contacto sexual, coerción, intento de violación y violación) el efecto del sexo, la tipología de adhesión al DES para libertad y recato sexual, y la interacción del sexo y la tipología de DES.

En primer lugar, se confirma la primera hipótesis planteada al observarse un efecto del sexo sobre la victimización y agresión sexual. Las mujeres informan mayor frecuencia de experiencias de victimización sexual en las categorías de contacto y coerción sexual 


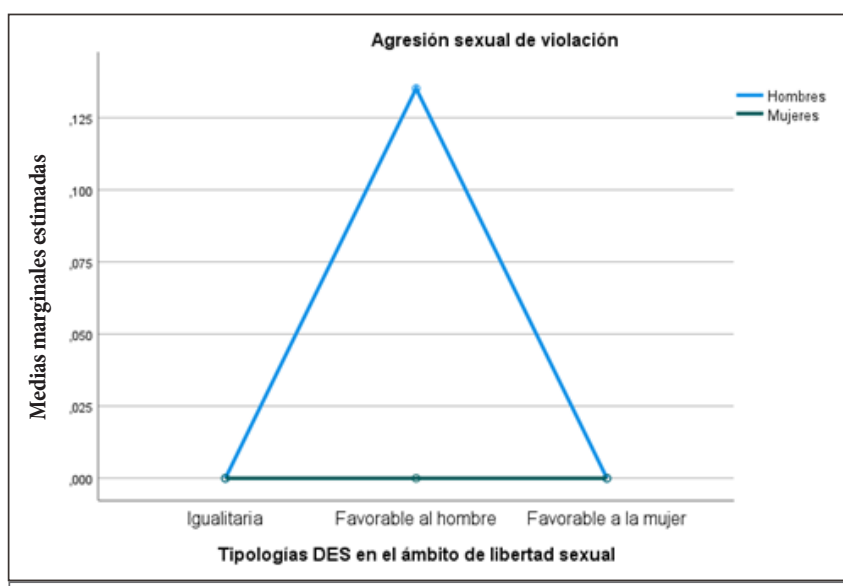

Figura 1: Efecto de interacción entre el sexo y la tipología DES en el ámbito de la libertad sexual sobre la agresión sexual de violación

en ambos ámbitos de los comportamientos sexuales (i.e., libertad y recato sexual), con un incremento de estas experiencias entre el 21\% y el $27.4 \%$ por el hecho de ser mujer. Por su parte, los hombres indican un mayor número de experiencias de agresión sexual en las categorías de intento de violación y violación en el ámbito de la libertad sexual, y en intento de violación en el ámbito del recato sexual, con un incremento entre el $12.4 \%$ al $13.9 \%$ asociado al hecho de ser hombre. Estos resultados se encuentran en consonancia con datos de prevalencia previos (Marcos et al., 2020; Ministerio del Interior, 2019; Oficina Europea de Estadística, 2021).

En segundo lugar, los análisis del efecto de la tipología de adhesión al DES confirman parcialmente la segunda hipótesis del estudio. Únicamente se observa un efecto de la tipología -tanto en el ámbito de la libertad como en el del recato sexual- para agresión sexual. Concretamente, este efecto se encuentra en la categoría de violación, con mayores experiencias de agresión en la tipología de adhesión al DES favorable al hombre en comparación con las tipologías favorable a la mujer e igualitaria, con un incremento entre el 10-10.4\% asociado al DES que favorece a los hombres. Es conocida la relación entre sexismo y violencia del hombre hacia la mujer (véase Juarros-Basterretxea et al., 2019). Específicamente, la vertiente tradicional del DES, entendida como mayor libertad sexual para el hombre que para la mujer, se ha asociado con actitudes a favor de



Figura 2: Efecto de interacción entre el sexo y la tipología DES en el ámbito del recato sexual sobre la agresión sexual de violación

la violación hacia la mujer (Jamshed \& Kamal, 2019; Lee et al., 2010; Mittal et al., 2017; Moyano et al., 2017; Sierra, Costa, \& Ortega, 2009; Sierra et al., 2010; Wanfield, 2018). Sin embargo, el metaanálisis de Endendijk et al. (2020) destacó la presencia del doble estándar sexual en relación a la victimización sexual. En concreto, este metaanálisis identificó la adhesión al DES que favorece a los hombres en víctimas de coerción sexual, y no así en el caso de los agresores (Endendijk et al, 2020). Esto nos lleva a pensar que se requiere más investigación en la relación de la actitud del DES con la agresión sexual.

Por su parte, con respecto a la interacción del sexo y la tipología de adhesión al DES, esta resulta significativa en la agresión en forma de violación, tanto en el ámbito de la libertad como en el del recato sexual. Los hombres con tipología favorable al hombre indican más experiencias de violación para agresión sexual. Este hallazgo confirma los resultados de la investigación previa sobre el DES que favorece a los hombres. Moyano et al. (2017) informaron de mayor adhesión al DES favorable a los hombres en chicos con experiencias de agresión sexual. Se sabe también que, en los hombres, la presencia del DES favorable a su género tiene capacidad para explicar la agresión sexual hacia las mujeres (Sierra, Gutiérrez-Quintanilla et al., 2009) y conduce a la aceptación de mitos hacia la violación (Lee et al., 2010). 
Este estudio aporta evidencias en el análisis del DES en el ámbito del recato sexual. El área del recato sexual adquiere especial relevancia, ya que el cambio de las sociedades hacia una postura más igualitaria y democrática podría conllevar nuevos guiones sexuales (Fasula et al., 2014; Suvivuo et al., 2010), como podría ser la defensa de una postura más conservadora (Sakaluk et al., 2014). Específicamente, en población española se ha observado mayor prevalencia de la tipología favorable al hombre en el ámbito del recato sexual que en el de la libertad sexual (Álvarez-Muelas et al., 2021). Por ello, es relevante considerar ambos ámbitos de comportamientos sexuales (i.e., libertad y recato sexual) a la hora de aborda el DES.

Este estudio no está exento de limitaciones. La más importante tiene que ver con la muestra empleada, seleccionada mediante muestreo incidental entre la población general, a través de redes sociales. Ello hace que las tasas de prevalencia de agresión y victimización sexual encontradas sean bajas. De hecho, la baja fiabilidad de consistencia interna del Sexual Experiences Scale obtenida en la muestra de este estudio probablemente sea consecuencia de ello. Además, los participantes son exclusivamente heterosexuales, por lo que futuros estudios deberían interesarse por otras orientaciones y minorías sexuales, tal como se viene sugiriendo en los últimos años (Calvillo et al., 2018, 2020). Todo ello limita la generalización de los resultados obtenidos.

En definitiva, la presente investigación contribuye al estudio de la agresión y victimización sexual. Se aportan evidencias acerca de las diferencias entre hombresy mujeres, observándose mayor número de experiencias de victimización sexual en las mujeres y de agresión sexual en los hombres. También, en congruencia con la investigación previa, destaca la relación de la tipología de adhesión al DES favorable al hombre con las experiencias de agresión sexual, siendo relevante analizar esta relación en los dos ámbitos actuales de DES: libertad y recato sexual. Por todo ello, se sugiere considerar el abordaje de los roles de género sobre los compartimientos sexuales en la prevención e intervención en las agresiones sexuales, considerándose imprescindibles actuaciones de prevención diversificadas, dada la multicausalidad de la conducta delictiva sexual (Redondo \& Mangot, 2017).

\section{- Conflicto de intereses}

Los autores declaran no tener conflictos de intereses.

\section{- Agradecimientos}

Esta investigación ha sido financiada por la Beca FPU 16/04429 de Formación de Profesorado Universitario como parte de la tesis de A.Á-M (Programa de Doctorado en Psicología B13 56 1; RD 99/2011).

\section{REFERENCIAS}

Álvarez-Muelas, A., Gómez-Berrocal, C., \& Sierra, J. C. (2020). Relación del doble estándar sexual con el funcionamiento sexual y las conductas sexuales de riesgo: Revisión sistemática [Relationship of sexual double standard with sexual functioning and risk sexual behaviors: A systematic review]. Revista Iberoamericana de Psicología y Salud, 11, 103-116. https:// doi.org/10.23923/i.rips.2020.02.038

Álvarez-Muelas, A., Gómez-Berrocal, C., \& Sierra, J. C. (2021). Typologies of sexual double standard adherence in Spanish population. European Journal of Psychology Applied to Legal Context, 13, 1-7. https:// doi.org/10.5093/eipalc2021a 1

Álvarez-Muelas, A., Gómez-Berrocal, C., Vallejo-Medina, P., \& Sierra, J. C. (2019). Invariance of Spanish version of Sexual Double Standard Scale across sex, age, and education level. Psicothema, 31, 465-474. https://doi.org/10.7334/ psicothema2019.102

Calvillo, C., Sánchez-Fuentes, M. M., ParrónCarreño, T., \& Sierra, J. C. (2020). Validation of the Interpersonal Exchange Model of Sexual Satisfaction Questionnaire in adults with a same-sex partner. International Journal of Clinical and Health Psychology, 20, 140-150. https://doi.org/10.1016/i. ijchp.2019.07.005 
Calvillo, C., Sánchez-Fuentes, M. M., \& Sierra, J. C. (2018). Revisión sistemática sobre la satisfacción sexual en parejas del mismo sexo [Systematic review on sexual satisfaction in same-sex couples]. Revista Iberoamericana de Psicología y Salud, 9(2), 115 -136. https:// doi.org/10.23923/i.rips.2018.02.018

Dunn, H. K., Gjelsvik, A., Pearlman, D. N, \& Clark, M. A. (2014). Association between sexual behaviors, bullying victimization and suicidal ideation in a national sample of high school students: Implications of a sexual double standard. Women's Health Issues, 24, 567-574. https://doi.org/10.1016/i. whi.2014.06.008

Endendijk, J. J., van Baar, A. L., \& Dekovi, M. (2020). He is a stud, she is a slut! A metaanalysis on the continued existence of sexual double standards. Personality and Social Psychology Review, 24, 163-190. https:// doi.org/10.1177/1088868319891310

European Union Agency for Fundamental Rights. (2014). Violence against women: An EU-wide survey. Results at a glance. Vienna, Austria: Oficina de Publicaciones de la Unión Europea. https://doi.org/10.2811/6080

Fandiño, R. Basanta, J., Sanmarco, J., Arce, R., \& Fariña, F. (2021). Evaluation of the executive functioning and psychological adjustment of child to parent offenders: Epidemiology and quantification of harm. Frontiers in Psychology, 12, 616855 . https:// doi.org/10.3389/fpsyg.2021.616855

Fasula, A. M., Carry, M., \& Miller, K. S. (2014). A multidimensional framework for the meanings of the sexual double standard and its application for the sexual health of young black women in the US. Journal of Sex Research, 51, 170-183. https://doi.org/ 10.1080/00224499.2012.716874

Garrido-Macías, M., Valor-Segura, I., \& Expósito, F. (2020). Which tactics of sexual violence I the relationship? The role of dependence towards partner. European Journal of Psychology Applied to Legal Context, 12, 5360. https://doi.org/10.5093/eipalc2020a6

Guo, Y. (2019). Sexual double standards in white and Asian Americans: Ethnicity, gender, and acculturation. Sexuality \& Culture, 23, 57-95. https://doi.org/10.1007/s12119. 018-9543-1

Jamshed, N., \& Kamal, A. (2019). Prevalence of rape myths and sexual double standards among university students in Pakistan. Journal of Interpersonal Violence, 36, 1-15. https:// doi.org/10.1177/0886260519844282

Juarros-Basterretxea, J., Overall, N., Herrero, J., \& Rodríguez-Díaz, F. J. (2019). Considering the Effect of Sexism on Psychological Intimate Partner Violence: A Study with Imprisoned Men. European Journal of Psychology Applied to Legal Context, 1 1, 61-69. https:// doi.org/10.5093/ejpalc2019a 1

Kim, Y., Lee, E., \& Lee, H. (2019). Sexual double standard, dating violence recognition, and sexual assertiveness among University Students in South Korean. Asian Nursing Research, 13, 47-52. https://doi. org/10.1016/i.anr.2019.01.003

Koon-Magnin, S., \& Ruback, R. B. (2012). Young adults' perceptions of nonforcible sexual activity: The effects of participant gender, respondent gender, and sexual act. Sex Roles, 67, 646-658. https://doi. org/10.1007/s $11199-012-0201-z$

Koss, M. P., Abbey, A., Campbell, R., Cook, S., Norris, J., Testa, M., \& White, J. (2007). Revising the SES: A collaborative process to improve assessment of sexual aggression and victimization. Psychology of Women Quarterly, 31, 357-370. https://doi. org/10.1111/i.1471-6402.2007.00385.x

Lee, J., Kim, J, \& Lim, H. (2010). Rape myth acceptance among Korean college students: The roles of gender, attitudes toward women, and Sexual Double Standard. Journal of Interpersonal Violence, 25, 1200-1223 https://doi. org/10.1177/0886260509340536

Malonda, E., Tur-Porcar, A., \& Llorca, A. (2017). Sexism in adolescence: Parenting styles, division of housework, prosocial behaviour and aggressive behaviour. Revista de Psicología Social, 32, 333-361. https:// doi.org/10.1080/02134748.2017.129174 $\underline{5}$ 
Marcos, V., Gancedo, Y., Castro, B., \& Selaya, A. (2020). Dating violence victimization, perceived gravity in dating violence behaviors, sexism, romantic love myths and emotional dependence between female and male adolescents. Revista Iberoamericana de Psicología y Salud, 1 1, 132-145. https:// doi.org/10.23923/i.rips.2020.02.040

Martínez-Gómez, A., Bolívar-Suárez J., ReyAnacona A., Ramírez-Ortiz C., LizarazoOjeda M., \& Yanez-Peñúñuri Y. (2021). Esquemas tradicionales de roles sexuales de género, poder en las relaciones y violencia en el noviazgo [Traditional schemes of sexual gender roles, power in relationships and dating violence]. Revista Iberoamericana de Psicología y Salud, 12, 1-16. https://doi. org/10.23923/i.rips.2021.01.041

Mayorga, E. G., Novo, M., Fariña, F., \& Arce, R. (2020). Destrezas cognitivas en menores infractores, de protección y normalizados: Un estudio de contraste [Cognitive skills in juvenile offenders, protection normalized youngsters: A contrastive study]. Revista Latinoamericana de Psicología, 52, 160-168 https://doi.org/10.14349/rlp.2020.v52.16

Ministerio del Interior. (2019). Informe sobre delitos contra la libertad e indemnidad sexual en España. Recuperado de http:// www.interior.gob.es/web/archivos-ydocumentacion/informe-sobre-delitoscontra-la-libertad-e-indemnidad-sexual-enespana

Mittal, S., Singh, T., \& Verma, S. (2017). Young adults' attitudes towards rape and rape victims: Effects of gender and social category. Journal of Psychology and Clinical Psychiatry, 7(4), 00447. https://doi.org/10.15406/ ¡рсру.2017.07.00447

Moyano, N., Monge, F. S., \& Sierra, J. C. (2017). Predictors of sexual aggression in adolescents: Gender dominance vs. rape supportive attitudes. European Journal of Psychology Applied to Legal Context, 9, 25-31. https://doi.org/10.1016/i. eipal.2016.06.001

Moyano, N., \& Sierra, J. C. (2014). Funcionamiento sexual en hombres y mujeres víctimas de abuso sexual en la infancia y en la adolescencia/adultez [Sexual functioning of men and women victims of childhood and adolescence/adulthood sexual abuse]. Revista Internacional de Andrología, 12, 132-138. $\quad$ https://doi.org/10.1016/i. androl.2014.04.012

Muehlenhard, C. L., \& Quackenbush, D. M. (2011). The Sexual Double Standard Scale. En T. D. Fisher, C. M. Davis, W. L. Yarber, R. Bauserman, G. E. Schree \& S. L. David (Eds.), Handbook of sexuality-related measures (pp. 199-200). New York, NY: Routledge.

Noriega, N., Juarros-Basterretxea, J. \& Herrero, J. (2020). Implicación de los profesionales de la salud en los casos de violencia en la pareja contra la mujer: La influencia de las actitudes sexistas hacia la mujer [Health professionals' involvement in cases of partner violence against women: The influence of sexist attitudes toward women]. Revista Iberoamericana de Psicologia y Salud. 11, 31-41. https://doi.org/10.23923/i. ríps.2020.01.033

Oficina Europea de Estadística. (2021). Homicidio intencional y delitos sexuales por estado legal y sexo de la persona involucrada - número y tasa para el grupo de sexo relevante. Recuperado de https:// ec.europa.eu/eurostat/databrowser/view/ crim hom soff/default/table?lang =en

Redondo, S., \& Mangot, Á. (2017). Génesis delictiva y tratamiento de los agresores sexuales: una revisión científica. Revista Electrónica de Ciencias Criminológicas, 2, 1-33. Recuperado de https://ojs. ehu.eus/index.php/eguzkilore/article/ download/18132/15699

Sakaluk, J. K., Todd, L. M., Milhausen, R., \& Lachowsky, N. J. (2014). Dominant heterosexual sexual scripts in emerging adulthood: Conceptualization and measurement. Journal of Sex Research, 51, 516-531. https://doi.org/10.1080/002244 99.2012.7454734

Sánchez-Fuentes, M. M., Moyano, N., GómezBerrocal, C., \& Sierra, J. C. (2020). Invariance of the Sexual Double Standard 
Scale: A cross-cultural study. International Journal of Environmental Research and Public Health, 17, 1569. https://doi.org/10.3390/ iierph 17051569

Sierra, J. C., Costa, N., \& Ortega, V. (2009). A validation study of the double standard scale and the rape supportive attitude scale in Brazilian women. International Journal of Psychological Research, 2, 90-98. https:// doi.org/10.21500/20112084.864

Sierra, J. C., Gutiérrez-Quintanilla, R., Bermúdez, M. P. \& Buela-Casal, G. (2009). Male sexual coerción: Analysis of a few associated factors. Psychological Reports, 105, 69-79. https://doi.org/10.2466/ PRO.105.1.69-79

Sierra J. C., Moyano, N., Vallejo-Medina, P., \& Gómez-Berrocal, C. (2018). An abridge Spanish version of Sexual Double Standard Scale: Factorial structure, reliability and validity evidence. International Journal of Clinical and Health Psychology, 18, 69-80. https:// doi.org/10.1016/i.ijchp.2017.05.003

Sierra, J. C., Santos-Iglesias, P., GutiérrezQuintanilla, R., Bermúdez, M. P., \& BuelaCasal, G. (2010). Factors associated with rape-supportive attitudes: Sociodemographic variables, aggressive personality and sexist attitudes. Spanish Journal of Psychology, 13, 202-209. https://doi.org/10.1017/ S1138741600003784

Sierra, J. C., Santos-Iglesias, P., Vallejo-Medina, P., \& Moyano, N. (2014). Autoinformes como instrumento de evaluación en sexología clínica. Madrid: Síntesis.
Stekhoven, D. J., \& Bühlmann, P. (2011). MissForest-non-parametric missing value imputation for mixed-type data. Bioinformatics, 28, 112-118. https://doi. org/10.1093/bioinformatics/btr597

Stevens, J. (1986). Applied multivariate statistics for social sciences. Hillsdale, NJ: LEA.

Suvivuo, P., Tossavainen, K., \& Kontula, $O$. (2010). "Can there be such a delightful feeling as this?". Variations of sexual scripts in Finnish girls' narratives. Journal of Adolescent Research, 25, 669-689. https:// doi.org/10.1177/0743558410366597

Teitelman, A. M., Tennille, J., Bohinski, J., Jemmott, L. S., \& Jemmott, J. B. (2013). Urban adolescent girls' perspectives on multiple partners in the context of the sexual double standard and intimate partner violence. Journal of the Association of Nurses in AIDS Care, 24, 308-321. https://doi. org/10.1016/i.jana.2013.04.001

Wanfield, S. M. (2018). "Men cannot be raped": Correlates of male rape myth acceptance. Journal of Interpersonal Violence, 36, 6391-6417. https://doi. org/10.1177/0886260518817777

Wilson, L., Mouilso, E., Gentile, B., Calhoun, K., \& Zeichner, A. (2015). How sexual aggression related to nonsexual aggression? A meta-analytic review. Aggression and Violent Behavior, 24, 199-213. https://doi. org/10.1016/i.avb.2015.04.010 